\title{
Wind Display Device for Locomotion Interface in a Virtual Environment
}

\author{
Sandip Kulkarni, Charles Fisher, Eric Pardyjak, Mark Minor and John Hollerbach \\ University of Utah, Salt Lake City, Utah 84112, USA
}

\begin{abstract}
This paper describes development of a wind display system for the TreadPort virtual environment locomotion interface, which is cumulatively known as the TreadPort Active Wind Tunnel (TPAWT). Computational Fluid Dynamic simulations and experiments with a scaled model test-bed of the system has resulted in a combination of passive and active controls capable of controlling wind speed and angle acting on a user in the environment. Development of the full scale TPAWT system is then described, which requires consideration of system geometry, integration, packaging, wind loads, and instrumentation.
\end{abstract}

KEYWORDS: flow control, computational fluid dynamics, haptic interface, virtual reality.

\section{INTRODUCTION}

The latest development of a new type of locomotion based virtual environment termed the TreadPort Active Wind Tunnel (TPAWT) is described. The TPAWT aims to improve the sense of immersion [1] created by the existing TreadPort Virtual Environment [2], Figure 1, by adding wind, olfactory, and radiant heat display. The existing TreadPort provides a rich CAVE-like visual interface [3] and accurate locomotion forces as a user walks through a virtual environment $[2,4,5]$. The TPAWT modifies this system by turning it into a controllable wind tunnel (the first of its kind) in order to regulate wind velocity (speed and orientation) acting on the user. These wind forces will be integrated with the graphical display $[6,7]$ and the Quick Urban Industrial Complex (QUIC) dispersion modeling system [8] to determine the wind flow patterns for improved totality and realism of the experience. QUIC then allows us to track odor dispersion and transport in the virtual environment, which will be recreated in the TPAWT by controlled injection of the scent particles into the wind tunnel.

The TreadPort Active Wind Tunnel is created by first enclosing the TreadPort, Fig. 1, with walls, floors, and ceilings with specific geometry such that air enters the environment from controllable vents adjoining the edge of the side screens, Figure 2. As we have shown with our scaled model of the system, the air streams merge at the front screen and then deflect to the user. We believe that this will provide a realistic sense of immersion to the user since it will appear as though the wind originates from the graphical environment

Relative to prior work, the Sensorama VR system in [9] considered inclusion of multi-modalities to improve immersion,

\author{
sandip.kulkarni@utah.edu \\ charlesjfisher@gmail.com \\ eric.pardyjak@utah.edu \\ mark.minor@utah.edu \\ jmh@cs.utah.edu
}

LEAVE 0.5 INCH SPACE AT BOTTOM OF LEFT COLUMN ON FIRST PAGE FOR COPYRIGHT BLOCK but confined the user to a seat. More recent VR systems developing wind interfaces, such as Wind Cubes [10] and blow displays [11], involve use of fans in proximity to the user which are more intrusive to the environment. Thus, our system is intended to remove these physical and optical intrusions while providing rich sensory experiences encountered while walking in the real world.

Our initial work on the TPAWT used Computational Fluid Dynamics (CFD) in order to evaluate design concepts. We then conducted experiments with a scaled model of the TPAWT to validate these concepts and evaluate our proposed control algorithms. This has been a highly iterative procedure where CFD simulations and physical experiments have been used to arrive at the scaled model test bed illustrated in Figure 2 and described later in this paper. With the scaled system, we proved that we could achieve control of wind speed and direction at the user position via a combination of passive and active control

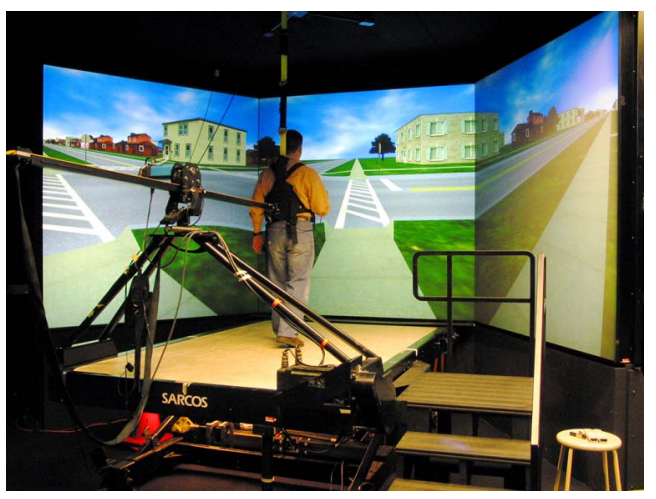

Figure 1 Treadport Virtual Environment comprising a CAVE-like visual display and locomotion interface

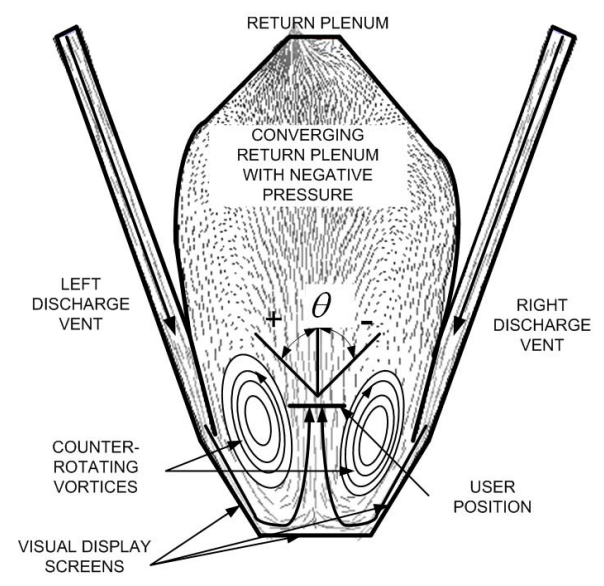

Figure 2 FLUENT simulation of wind flow in the plan view of scaled TreadPort Active Wind Tunnel showing vortices and the core flow at the user 
systems [12-14], which results in an entirely novel actively controlled wind tunnel. Previous research on adaptive wind tunnels [15-17] only considered open loop wall boundary adaptation to control wind speed. Thus, the TPAWT also contributes a new type of wind tunnel that allows real time control of localized wind flow patterns acting on a test specimen.

The description of the paper is as follows. Section 2 describes the design and development of the scaled model test bed, which has been used to validate the TPAWT concept, and presents new results considering a user in the system. In Section 3 we discuss extension of the scaled test bed for development of the full-scale system. This includes integration and packaging of air flow generation hardware, redesign of the CAVE-like display to accommodate wind loads, design of the walls of the returnplenum for flow stabilization, and sensor selection and placement. Finally, we discuss ongoing development and future work in Section 4. Section 5 provides concluding remarks.

\section{SCALE MODEL TEST BED}

In order to analyze wind flow in the system, we perform FLUENT CFD simulations on a 2D quarter scale model, Figure 2. A standard two-equation $\mathrm{k}-\varepsilon$ model is used for the simulations. The model geometry in Figure 2 is chosen after considering several alternatives such as [18] which proved infeasible. More recent results demonstrate that a different vent configuration improves controllability [ref first icra] which is further stabilized by a negative pressure return-plenum [14]. The FLUENT simulations were then used to develop quasi-steady input-output maps between vent velocities and wind velocity at the user [12].

\subsection{Control System}

The TPAWT requires the capability to control wind flow patterns, sustain the wind loads and provide the user a safe and non-intrusive environment within the confines of the existing system. This requires consideration of internal aerodynamics combined with active flow control methods to regulate wind flow at the user position.

Active flow control can be classified into three categories: 1) passive control $(\mathrm{PC}), 2)$ predetermined active (PDA) control, and 3) reactive feedback (RF) control [19]. Passive control involves guiding the flow with the aid of geometry. Predetermined active control of a fluid flow system involves energy input without feedback from the sensors. Reactive feedback control involves use of sensor feedback and actuators to control the flow in real-time.

The various components of the TPAWT system contribute PC,

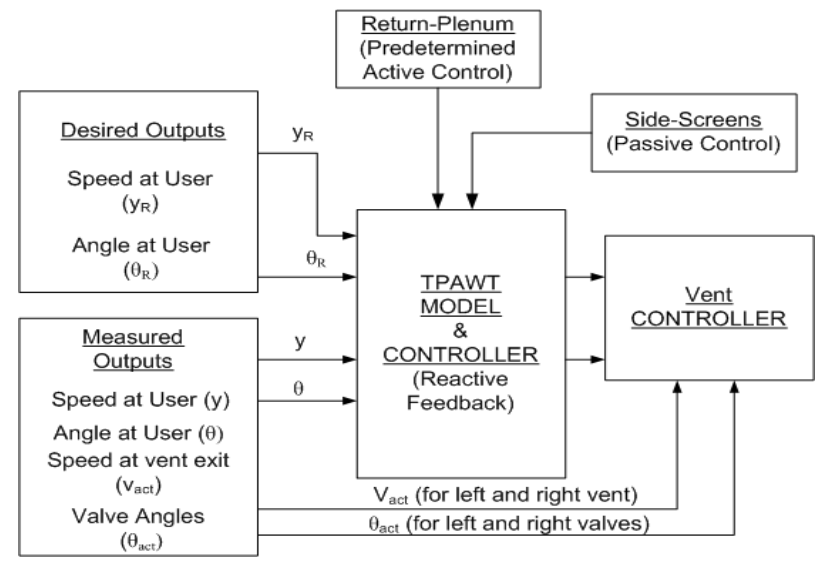

Figure 3 The block diagram of the integrated control system for the TPAWT
PDA control, and RF control methods. The visual display screens guide the air flow from the vents and contribute to the PC. The return-plenum is likewise essential for limiting the motion of the vortices and helps stabilize the flow. Since the negative pressure in the return-plenum draws air from the test-section, there is energy expenditure without regard to the sensors. This contributes to PDA control. The ducts and controllable vents along with the sensor are parts of the RF control.

The reactive feedback control requires regulation of the flow discharged by vents based upon sensor feedback. Since conservation of mass is observed, the net flow acting on the user is a function of the total bulk flow, $v_{T O T}$, entering in the system where $v_{T O T}=v_{L}+v_{R}$ and $v_{L}$ and $v_{R}$ are the left and right vent exit velocities, respectively. Vent velocities are then expressed as functions of total bulk flow velocity $\mathrm{v}_{T O T}$ and vent velocity ratio $\mathrm{v}_{L R}$, as given below:

$$
\begin{aligned}
& v_{L}=v_{T O T} \frac{v_{L R}}{\left(1+v_{L R}\right)}, \\
& v_{R}=v_{T O T} \frac{1}{\left(1+v_{L R}\right)} .
\end{aligned}
$$

where $v_{L R}=v_{L} / v_{R}$.

In [12-14, 20], the CFD based input-output maps are developed in terms of $v_{T O T}$ and $v_{L R}$. Wind speed at the user is mapped based upon $v_{T O T}$ and wind angle is mapped in terms of $v_{L R}$. [12] then details the development of an integrated controller using a dynamic extension that allows these static input-output maps to be used for dynamic control. The small gain theorem and discrete analysis are used to select control gains that assure stability of the closed loop system given uncertainty in the input-output maps.

Vortices on either side of the user dominate the flow response and several measures are used to account for their highly nonlinear dynamic response and interaction. A conditional angular rate switching controller is used to regulate wind angle at the user and essentially limits the rate of wind angle change. A flow conditioning slot is then used to further stabilize fluctuations in the air stream acting on the user. This also allows the air stream to approach the user from a much wider range of angles. Thus, our prior work demonstrates the capability of generating a wide range of wind speed and angles in the scaled model test bed.

Figure 3 shows the block diagram of the integrated system consisting of the various components of the TPAWT system. In

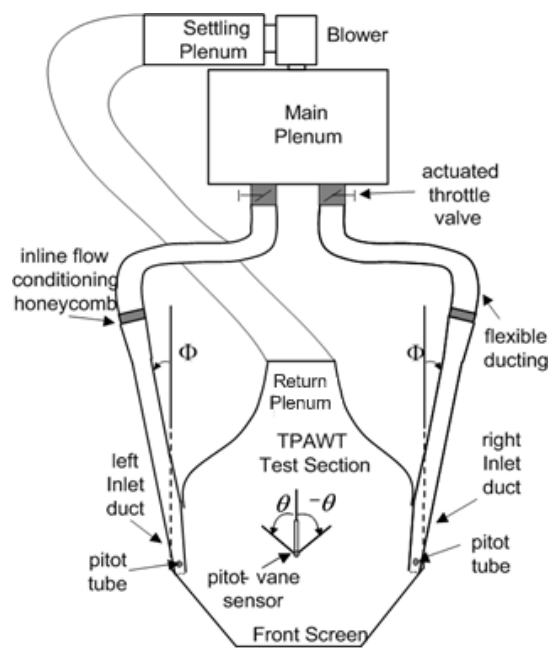

Figure 4: Scaled Test bed showing the vents, return-plenum, sensors and main plenum 
Figure $3, y_{R}$ and $\theta_{R}$ are the desired wind speed and angle at the user while $y$ and $\theta$ are the respective values measured by the pitot vane, Figure $5 . \mathrm{V}_{\text {act }}$ and $\theta_{\text {act }}$ are the vent speed and valve angle

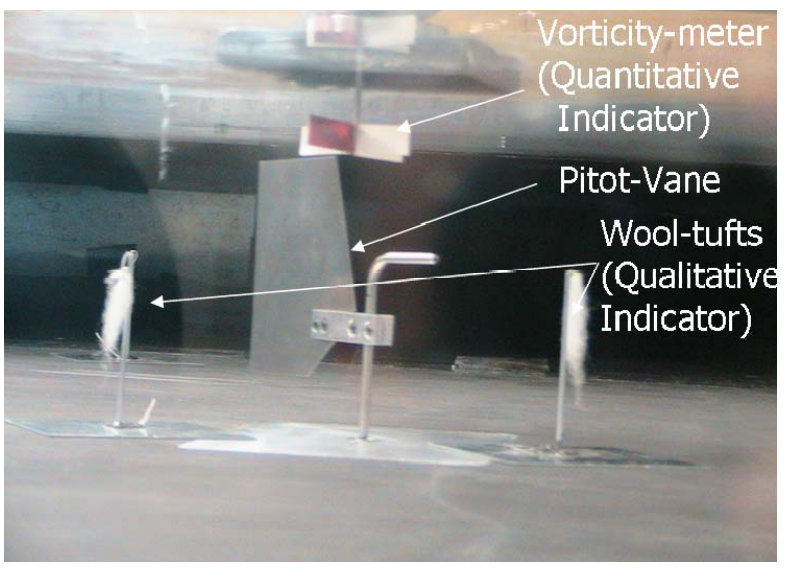

Figure 5: Pitot Vane, vorticity-meter and wool tufts showing the sensing system for $2 \mathrm{D}$ quarter-scaled test bed
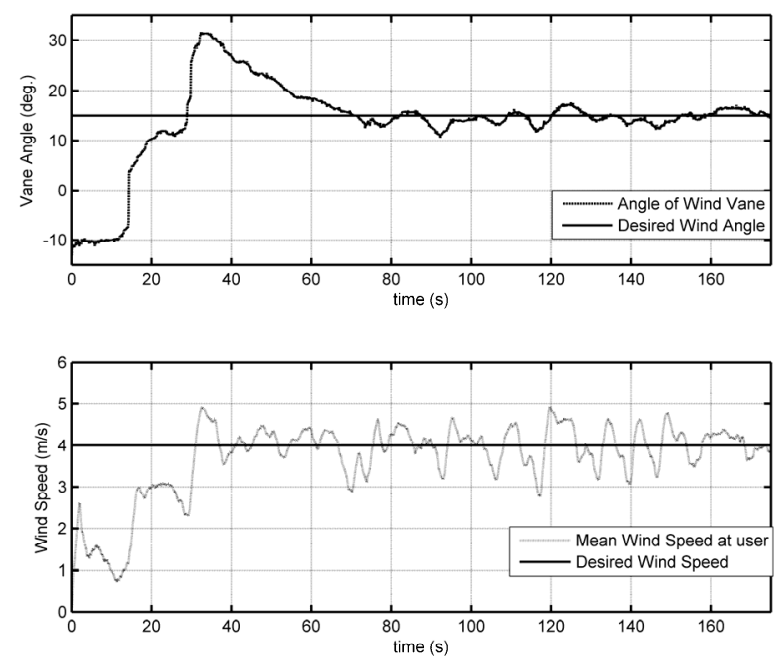

Figure 6: Wind Speed and Angle Control at the user position in the scaled test bed. measured by potentiometers and pitot probes. The required vent speed is determined from the output feedback controller [12-14, 20]. Vent speed is regulated by PI control loops that regulate the valve angles. Geared DC motors with anti-windup PI controllers assure that commanded valve angles are achieved.

\subsection{Experimental Hardware}

Figure 4 shows the plan view of the TPAWT scaled model test bed. The scale model incorporates actuated valves and a number of velocity sensors in order to facilitate real-time flow control. It is composed of a blower, a main plenum, return-plenum, butterfly throttling valves, ducting, and a test section. The settling plenum and blower draw air from the return plenum. The air received by the return plenum is fed back to the main plenum, forming a closed circuit as in Figure 4. Throttling valves are mounted on the side of the main plenum and are actuated by geared (66:1) Maxon DC motors $(20 \mathrm{~W})$. In order to approximate $2 \mathrm{D}$ flow, the gap height between floor and ceiling was reduced to $13 \mathrm{~cm}$ throughout the test section. The details of flow characterization are provided in [21].

The TPAWT test section has pitot tubes at each of the inlet ducts to determine vent velocities. A pitot-vane sensor, Figure 4 and Figure 5, mounted at the user position determines wind speed and direction at the user. The vane is designed to orient the pitot tube along the streamlines of the flow. A vorticity-meter [14], Figure 5, is mounted in the ceiling above the user position in order to detect proximity of the pitot-vane to the edge of the core flow. Dwyer 607-21 Differential Pressure Transmitters with a $250 \mathrm{~ms}$ response time are used to measure pitot tube pressure. Potentiometers measure valve angles and pitot-vane angle.

A dSpace 1103 Controller Board operating at a $1 \mathrm{kHz}$ sampling rate is used to control the experimental apparatus from Simulink using the Real Time Workshop toolbox.

\subsection{Wind Speed and Angle Control}

The control methods are implemented on the scaled model test bed after integrating the wind angle and speed controllers [12]. We are able to achieve simultaneous control of both wind speed and angle. Figure 6 shows wind speed and angle control at the user position when the integrated control system is applied to the scaled model test bed. Wind speed of $4 \mathrm{~m} / \mathrm{s}$ and a wind angle of $15^{\circ}$ can be observed in Figure 6 . Note that the delay in wind flow is the time required for the controller to overcome the strength of the stabilized vortices. Furthermore, sensor limitations due to noise in pitot probes, friction in the pitot vane and unsteadiness in flow also contribute to the delay and high variance of the wind

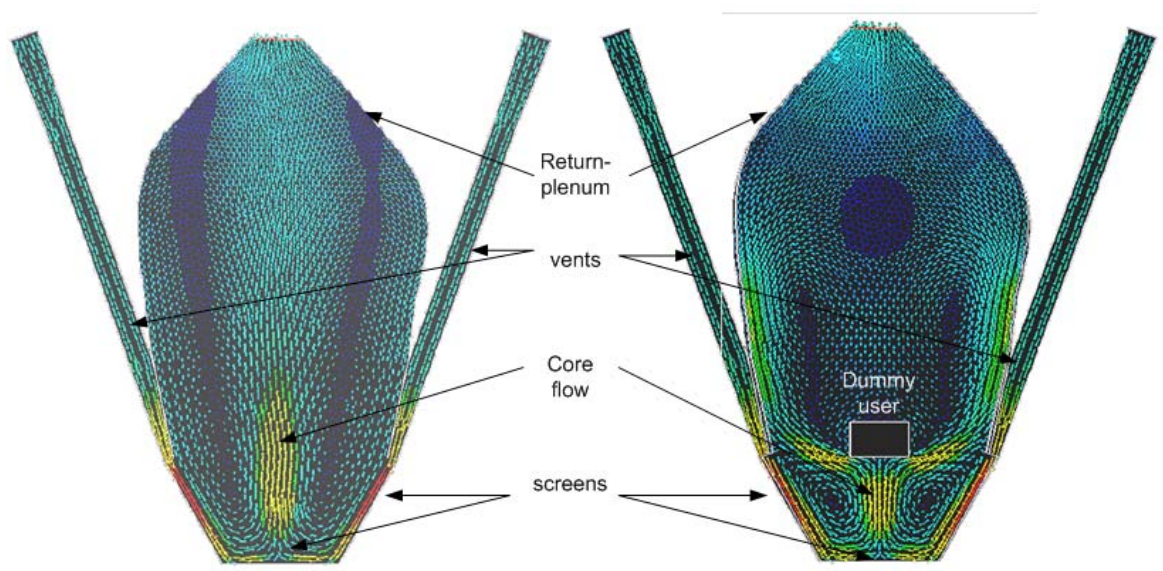

Figure 7: FLUENT simulation of 2D quarter-scaled TPAWT showing headwind and the effect of a dummy user on the core flow. 


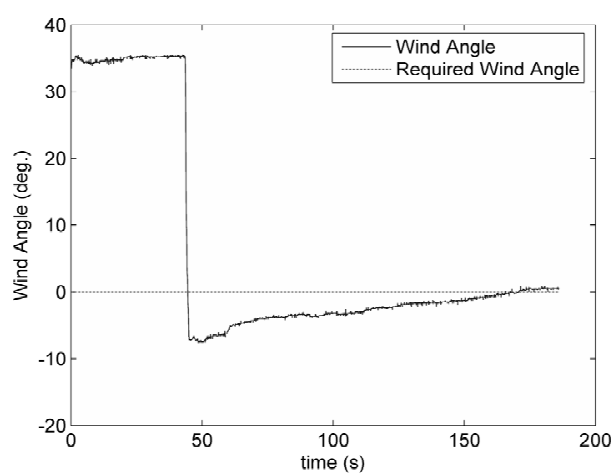

Figure 8 Wind Angle at the user position measured using pitot vane for $2 \mathrm{D}$ quarter-scaled model without a dummy object at the user position.

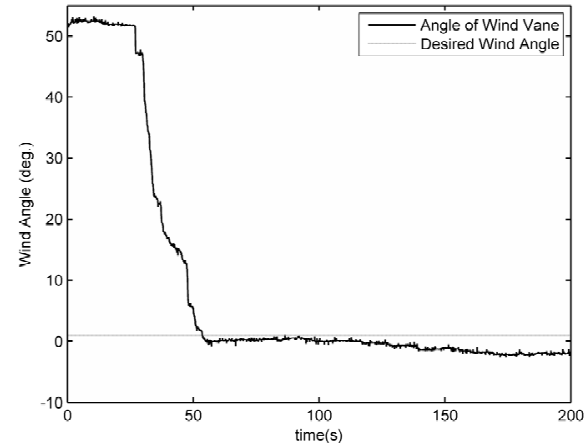

Figure 9 Wind Angle at the user position measured using pitot vane for $2 \mathrm{D}$ quarter-scaled model with a dummy object at the user position.

speed. We demonstrate in [12] that similar control of wind angles within $+/-30 \mathrm{deg}$, Table 1 , and with varying vent velocities is achieved.

\subsection{User in the environment:}

In previous studies $[12-14,18,20]$, the tests on scale model were performed without considering the user in the system. While ideal, this does not prove that the system will necessarily work as expected. Towards this goal, Figure 7 shows FLUENT CFD simulations of the wind flow in the scaled model system without a user and then with a scaled dummy block representing the user. It is important to note that the core flow is stable in both cases. The vortices are oblong without a user. In contrast, the vortices are well-defined and do not have enough room to destabilize with the user dummy block in the wind flow.

In order to evaluate the actual effect of the user on the air flow, we again use the scaled model test-bed to perform experiments with and without a dummy user. Figure 8 shows the wind angle at the user position without the dummy object where wind angle was again measured using the aforementioned pitot vane. Note that after an initial delay of about $40 \mathrm{~s}$, the wind angle was controlled to the desired value with an error of $\pm 3^{\circ}$ for 10 trials. Figure 9 shows the results for the experiment after including a dummy user behind the pitot vane. The results are actually similar to those without the user where the error in wind angle is again $\sim \pm 3^{\circ}$. Initial response and settling time, however, are actually much faster, which is attributed to the smaller and more contained vortices. Thus, we conclude that with small angles the user has
TABLE 1: PERFORMANCE OF VARIOUS WIND ANGLES

\begin{tabular}{|c|c|c|c|c|}
\hline Wind Angle & Delay & $\begin{array}{c}\text { Rise } \\
\text { Time }\end{array}$ & $\begin{array}{c}\text { Settling } \\
\text { Time }\end{array}$ & Error \\
\hline $30^{\circ}$ & $4 \mathrm{~s}$ & $95 \mathrm{~s}$ & $100 \mathrm{~s}$ & $1-2^{\circ}$ \\
\hline $20^{\circ}$ & $2-6 \mathrm{~s}$ & $44-48 \mathrm{~s}$ & $50 \mathrm{~s}$ & $1-3^{\circ}$ \\
\hline $10^{\circ}$ & $15 \mathrm{~s}$ & $40 \mathrm{~s}$ & $55 \mathrm{~s}$ & $1-2^{\circ}$ \\
\hline $0^{\circ}$ & $25 \mathrm{~s}$ & $20 \mathrm{~s}$ & $45 \mathrm{~s}$ & $1-3^{\circ}$ \\
\hline$-10^{\circ}$ & $2 \mathrm{~s}$ & $46 \mathrm{~s}$ & $50 \mathrm{~s}$ & $2-4^{\circ}$ \\
\hline$-20^{\circ}$ & $2-15 \mathrm{~s}$ & $5-7 \mathrm{~s}$ & $45-50 \mathrm{~s}$ & $2-5^{\circ}$ \\
\hline$-30^{\circ}$ & $1-12 \mathrm{~s}$ & $2-20 \mathrm{~s}$ & $45 \mathrm{~s}$ & $1-2^{\circ}$ \\
\hline
\end{tabular}

minimal effect on the accuracy of wind angle and actually seems to improve time response.

\section{FULL-SCALE TPAWT}

We now present development of the full scale TPAWT system. Results from the scaled model have been critical for packaging the system, creating its geometry, considering wind loads, and for selecting sensors, all of which are now described.

\subsection{Packaging and Integration}

One of the biggest challenges in development of full scale system is packaging of all of the components with sufficient space for conditioning the air flow such that it is uniform at the vent outlet and similar to $2 \mathrm{D}$ scale model. Figure 10 shows a solid model of the full-scale system. This model includes the controllable vents, ducting, return plenum with filters, fan, and decking along with the visual screens and the treadport. Figure 11 shows the actual assemblage of those parts into the treadport

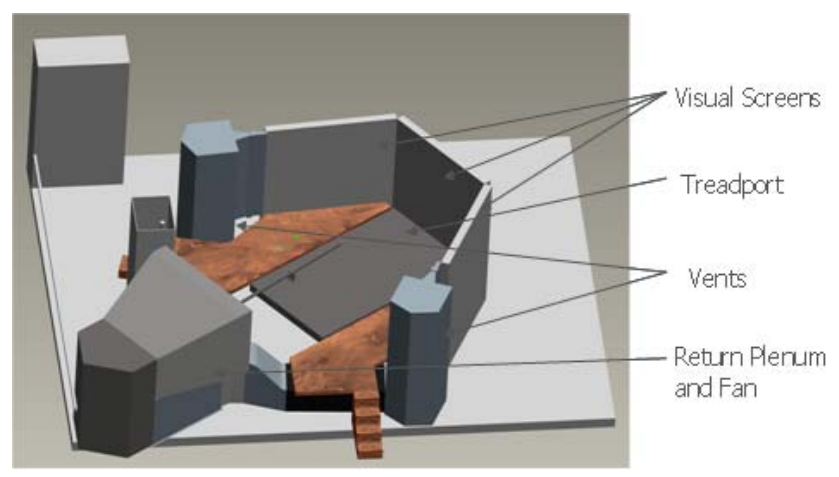

Figure 10: Solid model showing the TreadPort Active Wind Tunnel with various parts.

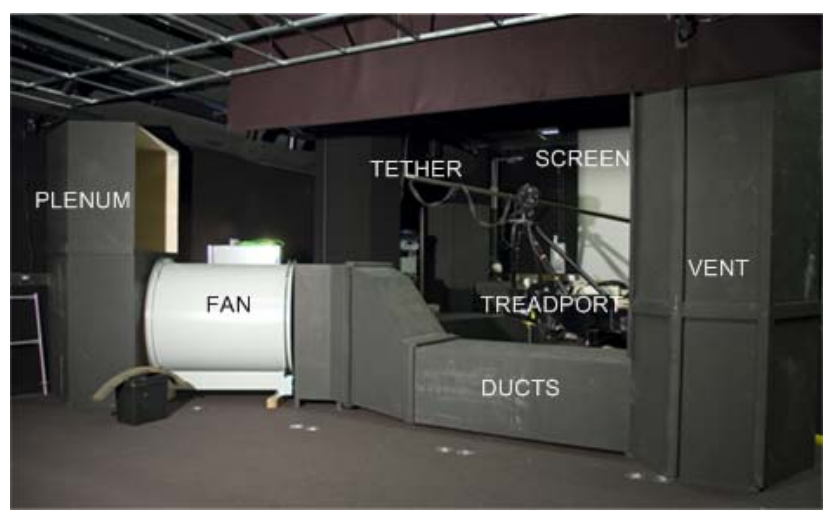

Figure 11 Full-scale TreadPort Active Wind Tunnel while under construction. 


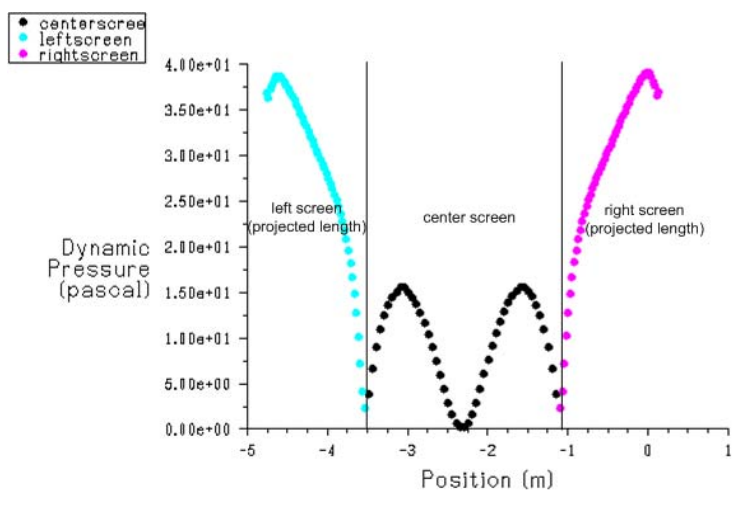

Figure 12 Pressure profile showing dynamic pressure acting on the visual display screens of 2D TPAWT
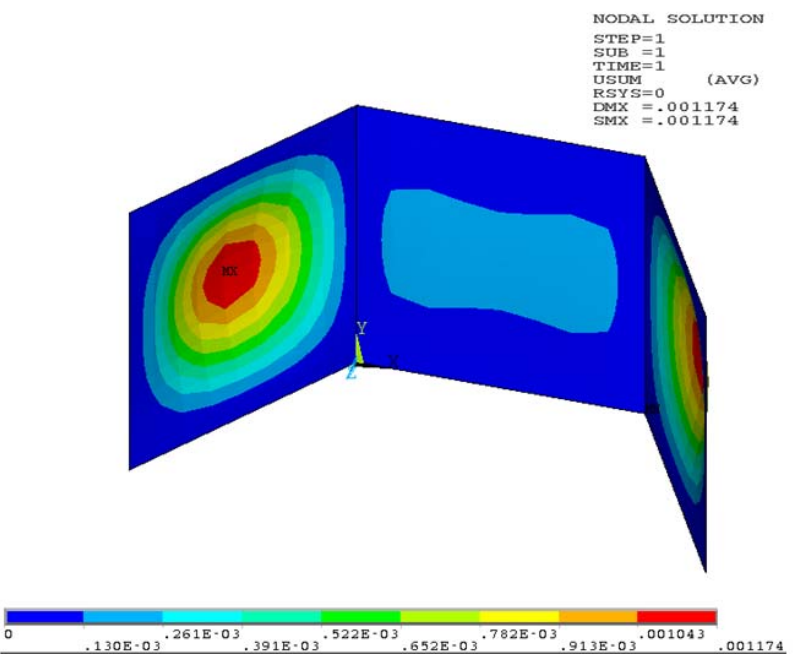

Figure 13 Resultant displacement of the screens resulting from the dynamic pressure of the wind on the redesigned screens with $3 / 4$ " screens and modified boundary conditions

room.

Notice that ducts are perfectly symmetric to improve uniformity of the flow and they are routed below the decking to minimize intrusion. Valves controlling vent flow are positioned as close to the vents as possible in order to minimize time delay associated with the air flow while also leaving sufficient room for flow conditioning hardware in the vent tower to assure uniform vent output. Also note that the fan, a 45HP vane-axial unit, was selected for its high flow rate and extremely quiet operation. This has allowed the fan to become an integral part of the TPAWT without intruding on the immersion of the system.

We also improve sensing by replacing the pitot vane with a $2 \mathrm{D}$ ultrasonic anemometer, Model 85000, from RM Young Company. This sensor reduces noise, eliminates frictional nonlinearities of pitot vane, and we expect will provide improved response time. The sensor is larger, though, and in order to make it non-intrusive to the user, we place it on the ceiling above the user position. Furthermore, it has a wind speed accuracy of $0.1 \mathrm{~m} / \mathrm{s}$, wind directional accuracy of $\pm 2^{\circ}$, and it can measure low velocities with much more reliability.

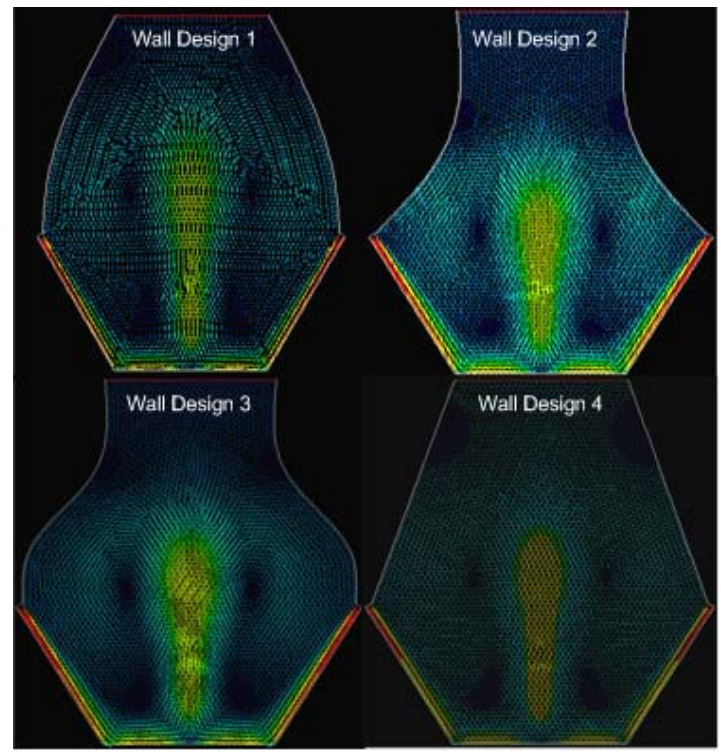

Figure 14 FLUENT simulations for various wall designs showing stable core flow for vent inlet velocities of $6 \mathrm{~m} / \mathrm{s}$ for the left and right vents.

\subsection{Wind Loading}

While the air pressure in the system is relatively small, the large area of the display screens results in modest forces. Unfortunately, the original screens were only intended for providing immersion and are only 0.25 "thick. In order to analyze their deflection, we perform 2D FLUENT CFD simulations of the full scale system to predict nominal pressures acting on the screens, Figure 12. As result, we estimate that a pressure of $\sim 40$ $\mathrm{Pa}$ acts on the side screens when the vent inlet speed is $6 \mathrm{~m} / \mathrm{s}$ for the left and right vents. It is also important to note that the $2 \mathrm{D}$ simulation used here provides conservative estimates for the wind loading.

A finite element analysis using ANSYS for the preexisting display screens and its boundary conditions shows that the screens deflect by about $\sim 30 \mathrm{~cm}$, given the aforementioned loads. In order to reduce such a high deflection, we perform several design iterations by changing boundary conditions and screen thickness. We improve the structural design by constraining all the boundary edges of the three screens, consider fastening of the three screens, and increase the thickness of screens to $3 / 4$ ". The resulting finite element analysis, Figure 13, shows that the deflection of screens reduces to $\sim 1.1 \mathrm{~mm}$. Visual experiments on the preexisting screens show that deflection up to $3 \mathrm{~mm}$ cannot be easily noticed by the user. Hence, the new screen design is within acceptable limits.

\subsection{Return Plenum Wall Design}

The user position is aligned with the center of the front screen and the outlet. In order to constrain the core flow towards the user, the return-plenum walls are designed to act as a nozzle. The geometry of the walls restricts the motion of the vortices and stabilizes the flow. FLUENT simulations for various wall designs show that the stability of core flow is not affected by the shape of the nozzle formed by return-plenum walls as long as the returnplenum acts as a nozzle, Figure 14. Considering the ease of manufacturing, we use the straight wall design in Figure 14 (4). 


\section{4}

While the research presented here is quite promising, quite a number of issues remain to be addressed. While we have shown that a user in the environment is actually beneficial for small wind angles, we still need to evaluate this over a larger range. Likewise, we have presented overall design of full scale TPAWT system, but again many details remain. Reinforcement of the frame supporting the screens must be considered and design and fabrication of the ceiling and decking must be completed. Furthermore, characterization of the full scale system must be repeated similar to the scaled model system.

We are also in the process of integrating Graphics Processing Units (GPU) to simulate wind flow and particle dispersion in urban areas based upon the QUIC model $[6,7]$. While these wind flows serve as input to the wind generation system, they are also presented in the graphical display to present subtle effects such as leaves blowing and trees swaying. Ongoing work also involves inclusion of olfactory display and radiant heat effects into the system. We expect that this cumulatively provide a rich multimodal virtual experience. Future work will focus on psycho physical experiments, use of the system for emergency response training, and for gait therapy rehabilitation.

\section{CONCLUSION}

Ongoing research on wind display in locomotion based virtual environments is discussed. This paper demonstrates that users in the wind flow can actually be beneficial to system response and describes how the scaled model test bed is used for design of the full scale TPAWT system. Issues related to system integration and packaging are considered. Important aspects of valve placement and system symmetry are presented. Models of wind loads acting on the CAVE-like screens are also presented and solutions for remediating excessive screen displacement are provided.

\section{ACKNOWLEDGEMENT}

This research is supported by NSF Grant ISS-0428856. The authors would like to thank Mark Deaver, Aditya Desai, and Shanthanu Chakravarthy for their assistance on the scaled model and full scale systems.

\section{REFERENCES}

[1] H. Q. Dinh, N. Walker, C. Song, A. Kobayashi, and L. F. Hodges, "Evaluating the Importance of Multi-sensory Input on Memory and the Sense of Presence in Virtual Environments," Proceedings of the IEEE Virtual Reality, p. 222, 1999.

[2] J.M.Hollerbach, Y. Xu, R.Christensen, and S.C.Jacobsen, "Design specifications for the second generation Sarcos Treadport locomotion interface," in Haptics Symposium,Proceedings of ASME Dynamic Systems and Control Division. vol. 69-2 Orlando, 2000, pp. 1293-1298.

[3] C. Cruz-Neira, D. Sandin, and T. A. DeFanti, "Surround-screen projection-based virtual reality: the design and implementation of the CAVE," in ACM SIGGRAPH, Anaheim, CA, Aug. 1993, pp. 135-142.

[4] J.M Hollerbach, "Locomotion interfaces," in Handbook of Virtual Environments: Design, Implementation, and Applications, e. K.M Stanley, Ed.: Lawrence Erlbaum Associates, Inc., 2002, pp. 239-254.

[5] D. I. Grow and J. M. Hollerbach, "Harness design and coupling stiffness for two-axis torso haptics," in 14th Symp. Haptic Interfaces for Virtual Environments and Teleoperator Systems, Arlington, VA, 2006.

[6] P. Willemsen, A. Norgren, B. Singh, and E. R. Pardyjak, "'Development of a new methodology for improving urban fast response Lagrangian dispersion simulation via parallelism on the graphics processing unit," " in Proc. 11th Intl. Conf. on Harmonisation within Atomspheric Dispersion Modelling for Regulatory Purposes Queen's College, University of Cambridge, U.K., 2007.

[7] E. R. Pardyjak, B. Singh, A. Norgren, and P. Willemsen, "'Using video gaming technology to achieve low-cost speed up of emergency response urban dispersion simulations,"" in 7th Symp. on the Urban Environment, American Meteorological Society San Diego, 2007.

[8] E. R. Pardyjak and M. J. Brown, "Evaluation of a fast-response urban wind model comparison to single-building wind-tunnel data.," in Proceedings of the 2001 International Symposium on Environmental Hydraulics Tempe, AZ, 2001.

[9] M. H. Morton, "Sensorama," U. S. Patent, Ed., 1961.

[10] T. Moon and G. J. Kim, "Design and Evaluation of a Wind Display for Virtual Reality," in 11th ACM Symposium on Virtual Reality Software and Technology, VRST 2004, Hong Kong, China, Hong Kong, 2004, pp. p 122-128.

[11] M. Minakuchi and S. Nakamura, "Collaborative Ambient Systems by Blow Displays," in Tangible and Embedded Interactions, Baton Rouge, Lousiana, 2007, pp. 105-108.

[12] S. D. Kulkarni, M. A. Minor, M. W. Deaver, E. R. Pardyjak, and J. M. Hollerbach, "Control of Wind Flow Patterns in a Virtual Environment," IEEE Control System Technology, vol. (submitted), 2008. [13] S. D. Kulkarni, M. A. Minor, M. W. Deaver, and E. R. Pardyjak, "Output feedback control of wind display in a virtual environment," in Proc. IEEE Intl. Conf. Robotics and Automation, Rome, Italy, 2007, pp. $832-839$.

[14] S. D. Kulkarni, M. A. Minor, M. W. Deaver, E. R. Pardyjak, and J. M. Hollerbach, "Steady Headwind Display with Conditional Angular Rate-Switching Control," in Proc. IEEE Intl. Conf. Robotics and Automation, Pasadena, California, 2008

[15] W. R. Sears, "SELF CORRECTING WIND TUNNELS," Aeronautical Journal, vol. 78, pp. 80-89, 1974.

[16] W. R. Sears, R. J. Vidal, J. C. Erickson, Jr., and A. Ritter, "INTERFERENCE-FREE WIND-TUNNEL FLOWS BY ADAPTIVEWALL TECHNOLOGY," Journal of Aircraft, vol. 14, pp. 1042-1050, 1977.

[17] S. Wolf, "Adaptive wall technology for improved wind tunnel testing techniques-a review," Progress in Aerospace Sciences, vol. 31, pp. 85136, 1995.

[18] R. Kirkman and M. Metzger, "Conceptual Design of an adaptive wind tunnel for the generation of an unsteady complex flow patterns," in ASME 2005 Fluids Engineering Division Summer Meeting and Exhibition, Houston, Texas, June 19-23 2005.

[19] M. Gad-el-Hak., Flow control : passive, active, and reactive flow management. Cambridge, New York: Cambridge University Press, 2000.

[20] S. D. Kulkarni, M. A. Minor, E. R. Pardyjak, and J. M. Hollerbach, "Combined Wind Speed and Angle Control in a Virtual Environment," in International Conference on Intelligent Robots and Systems, Nice, France, 2008.

[21] M. Deaver, "Creating Wind Display for a Virtual Environment," in Mechanical Engineering. vol. Master of Science Salt Lake City: Utah, December 2007, p. 86. 